\title{
Energía Solar fotovoltaica: Medida de las curvas características de un módulo fotovoltaico utilizando un trazador de curvas I-V a temperatura constante y a irradiación solar constante tomando como base la norma IEC 60904-1:2020
}

Photovoltaic Solar Energy: Measurement of the typical curve of a photovoltaic module with an I-V curve tracer at constant temperature and constant solar irradiation based on the

${ }^{1}$ Docente Materia Resistencia de Materiales. Universidad Privada del Valle. Sucre. Bolivia. juanalfredotorrico@gmail.com

${ }^{2}$ Ingeniero civil. Máster en Energías Renovables. Universidad de Salamanca. Salamanca. España.mariagullonurbano@hotmail.com

\section{RESUMEN}

El presente trabajo de investigación representa el primero de una serie de ensayos, con módulos fotovoltaicos que han sido desarrollados en el Centro de Investigaciones energéticas, medioambientales y tecnológicas (C.I.E.M.A.T.), en la ciudad de Madrid, España. Un módulo fotovoltaico (FV) viene definido eléctricamente por su curva de intensidad-voltaje (I-V). Esta curva depende básicamente de la irradiancia solar incidente. El presente estudio consistió en la medición de las curvas I-V para un módulo fotovoltaico de silicio monocristalino utilizando como herramienta el trazador de curvas I-V "PVPM 1000 CX", donde el módulo FV se midió a varias condiciones de laboratorio, encontradas en la etapa de operación: a) manteniendo una radiación solar constante de $857 \mathrm{~W} / \mathrm{m}^{2}$ en el módulo FV para cinco temperaturas diferentes de $38^{\circ} \mathrm{C}, 40{ }^{\circ} \mathrm{C}, 44,9{ }^{\circ} \mathrm{C}, 49,65^{\circ} \mathrm{C}$ y $53,4{ }^{\circ} \mathrm{C}$; y b) manteniendo una temperatura constante de 51,4 


\title{
JOURNAL BOLIVIANO DE CIENCIAS - Vol. 17 - Número 50
}

ISSN: $2075-8944$

Universidad Privada del Valle - Bolivia

https://doi.org/10.52428/20758944.v17i50.24

${ }^{\circ} \mathrm{C}$ en el módulo para cuatro radiaciones solares diferentes de $857 \mathrm{~W} / \mathrm{m}^{2}, 596 \mathrm{~W} / \mathrm{m}^{2}, 727 \mathrm{~W} / \mathrm{m}^{2}$ y $784 \mathrm{~W} / \mathrm{m}^{2}$.

Se han elaborado las curvas I-V, se ha analizado el comportamiento del módulo con las condiciones impuestas en lo que respecta a la tensión de circuito abierto $\left(\mathrm{V}_{\mathrm{oc}}\right)$ y la intensidad de corto circuito $\left(\mathrm{I}_{\mathrm{sc}}\right)$. Los resultados obtenidos de las mediciones reproducen la tradicional forma de las curvas I-V para las nueve condiciones de laboratorio, también se demuestra que en ninguno de los ensayos se ha sobrepasado los valores de condiciones estándar $\left(\mathrm{I}_{\mathrm{sc}}\right)$ y $\left(\mathrm{V}_{\mathrm{oc}}\right)$ del módulo fotovoltaico.

Palabras clave: Curva I-V. Energía solar Fotovoltaica. Irradiancia. I $\mathrm{I}_{\mathrm{sc}}$. Módulo-siliciomonocristalino. Trazador de curvas PVPM 1000CX. Voc.

\begin{abstract}
This research work represents the first of a series of tests with photovoltaic modules which have been developed at the Center for Energy, Environmental and Technological Research (C.I.E.M.A.T.), in the city of Madrid, Spain. A photovoltaic (PV) module is electrically defined by its current-voltage (I-V) curve. This curve basically depends on the incident solar irradiance. The present study consisted on measuring the IV curves for a monocrystalline silicon photovoltaic module using the IV curve tracer "PVPM 1000 CX" as a tool, where the PV module was measured at various laboratory conditions, found in the operation stage: a) maintaining a constant solar radiation of $857 \mathrm{~W} / \mathrm{m}^{2}$ in the PV module for five different temperatures of $38{ }^{\circ} \mathrm{C}$, $40{ }^{\circ} \mathrm{C}, 44.9{ }^{\circ} \mathrm{C}, 49.65{ }^{\circ} \mathrm{C}$ and $53.4{ }^{\circ} \mathrm{C}$; and b) maintaining a constant temperature of $51.4{ }^{\circ} \mathrm{C}$ in the module for four different solar radiations of $857 \mathrm{~W} / \mathrm{m}^{2}, 596 \mathrm{~W} / \mathrm{m}^{2}, 727 \mathrm{~W} / \mathrm{m}^{2}$ and $784 \mathrm{~W} / \mathrm{m}^{2}$.
\end{abstract}

The I-V curves have been drawn up, the behavior of the module has been analyzed with the conditions imposed regarding the open circuit voltage $\left(\mathrm{V}_{\mathrm{oc}}\right)$ and the short circuit intensity $\left(\mathrm{I}_{\mathrm{sc}}\right)$. The results obtained from the measurements reproduce the traditional shape of the IV curves for the nine laboratory conditions, it is also shown that, in none of the tests, the values of standard conditions $\left(\mathrm{I}_{\mathrm{sc}}\right)$ and $\left(\mathrm{V}_{\mathrm{oc}}\right)$ of the photovoltaic module have been exceeded. 
Keywords: I-V curve. Photovoltaic solar energy. Irradiance. $I_{\mathrm{sc}}$. Monocrystalline silicon module. PVPM 1000 CX curve tracer. $\mathrm{V}_{\text {oc. }}$.

\section{INTRODUCCIÓN}

La energía solar ofrece un enorme potencial energético que puede aprovecharse mediante diversas tecnologías. Una de ellas es la energía solar fotovoltaica (FV). Dada la disponibilidad global de la energía solar, esta tecnología es una solución alternativa para generar electricidad mediante conexiones a redes de suministro locales y autónomas.

Es ampliamente conocido, dentro del campo de la energía solar fotovoltaica, que alrededor de un $84 \%$ de la energía solar incidente en un módulo fotovoltaico (también llamado panel o placa) ordinario de silicio monocristalino, se transforma en calor; y el 16\% restante se transforma en electricidad (Abella, 2001), esto solo se consigue operar en el punto de máxima potencia (MPP). Para un correcto entendimiento de lo indicado, se debe cuantificar la electricidad; tensión (el voltaje de salida disminuye con la temperatura del orden de $2.3 \mathrm{mV} /{ }^{\circ} \mathrm{C}$ para silicio monocristalino); intensidad de corriente (la corriente generada es directamente proporcional a la irradiancia) y la potencia que son capaces de generar los módulos fotovoltaicos (Collado, 2015). Sin embargo, toda esta información se encuentra codificada en la gráfica denominada curva tensión-intensidad I-V.

También influyen de forma importante, aunque en menor grado, otros factores como la ubicación geográfica (que tiene relación con el ángulo de incidencia de la radiación solar sobre el generador fotovoltaico), la respuesta espectral (el espectro solar varía con la hora del día, la estación del año) que, en el caso de las células, es diferente para cada fabricante y tecnología.

La elaboración de la curva característica de un módulo fotovoltaico, también llamada curva I-V de corriente y voltaje, es fundamental para analizar su desempeño eléctrico (Krenzinger et al., 2009). Con la curva I-V es posible obtener parámetros importantes respecto al comportamiento eléctrico del módulo como ser la corriente de cortocircuito $\left(\mathrm{I}_{\mathrm{sc}}\right)$, voltaje de circuito abierto $\left(\mathrm{V}_{\mathrm{oc}}\right)$ 


\section{JOURNAL BOLIVIANO DE CIENCIAS - Vol. 17 - Número 50}

ISSN: $2075-8944$

Universidad Privada del Valle - Bolivia

https://doi.org/10.52428/20758944.v17i50.24

y el punto de máxima potencia (MPP). De acuerdo con la norma de referencia IEC 60904-1:2020 (International Electrotechnical Commision [IEC], 2020), la determinación de esta curva debe ser realizada dentro de las condiciones estándar de temperatura y radiación solar $\mathrm{G}=1000 \mathrm{~W} / \mathrm{m}^{2}$ y $\mathrm{T}_{\mathrm{c}}=25^{\circ} \mathrm{C}$.

En las condiciones reales de trabajo, un módulo fotovoltaico recibirá una irradiancia menor a $1000 \mathrm{~W} / \mathrm{m}^{2}$. Por ejemplo, cuando los rayos solares no infiltran perpendicularmente a su superficie o también cuando el cielo se cubra.

El presente estudio se centra en el aspecto descrito anteriormente. Someter un módulo fotovoltaico a diferentes condiciones experimentales de trabajo y medir las curvas I-V para estas condiciones mediante el dispositivo PVPM 1000CX. Se ha seleccionado un módulo de silicio monocristalino modelo SUNTECH STP 175S-24/Ac para ensayarlo a cuatro mediciones de radiación solar manteniendo una temperatura constante de $51,4{ }^{\circ} \mathrm{C}$ en el módulo $\mathrm{FV}$, y cinco mediciones de temperaturas en el panel manteniendo una radiación solar constante de $857 \mathrm{~W} / \mathrm{m}^{2}$.

Debido a que no es posible manipular fácilmente estos parámetros de medición definidos, se han empleado varios días para realizar los ensayos de la presente investigación. Dentro de la revisión a las normas de ensayo de módulos fotovoltaicos para elaborar el presente trabajo, se optó por la norma de referencia: IEC 60904-1:2020, Photovoltaic devices - Part 1: Measurements of photovoltaic current-voltage characteristics (IEC, 2020). Esta norma describe los procedimientos para la medición de las curvas características I-V de los dispositivos fotovoltaicos sometidos a luz solar natural o simulada. Estos procedimientos son aplicables a una célula solar, a un subconjunto de células solares, o a todo el módulo FV.

\section{METODOLOGÍA}

\subsection{Lugar de experimentación y materiales utilizados}

El trabajo de investigación ha sido realizado en la azotea del edificio 42 (Laboratorio de Fotovoltaica) del Centro de Investigaciones energéticas, medioambientales y tecnológicas (C.I.E.M.A.T.). A continuación, se describen los equipos y dispositivos elegidos: 
a) Módulo fotovoltaico: Se trata de un módulo de tecnología china compuesto por 72 células monocristalinas en serie de alta potencia con una eficiencia de hasta el $14.1 \%$ (Figura 1).
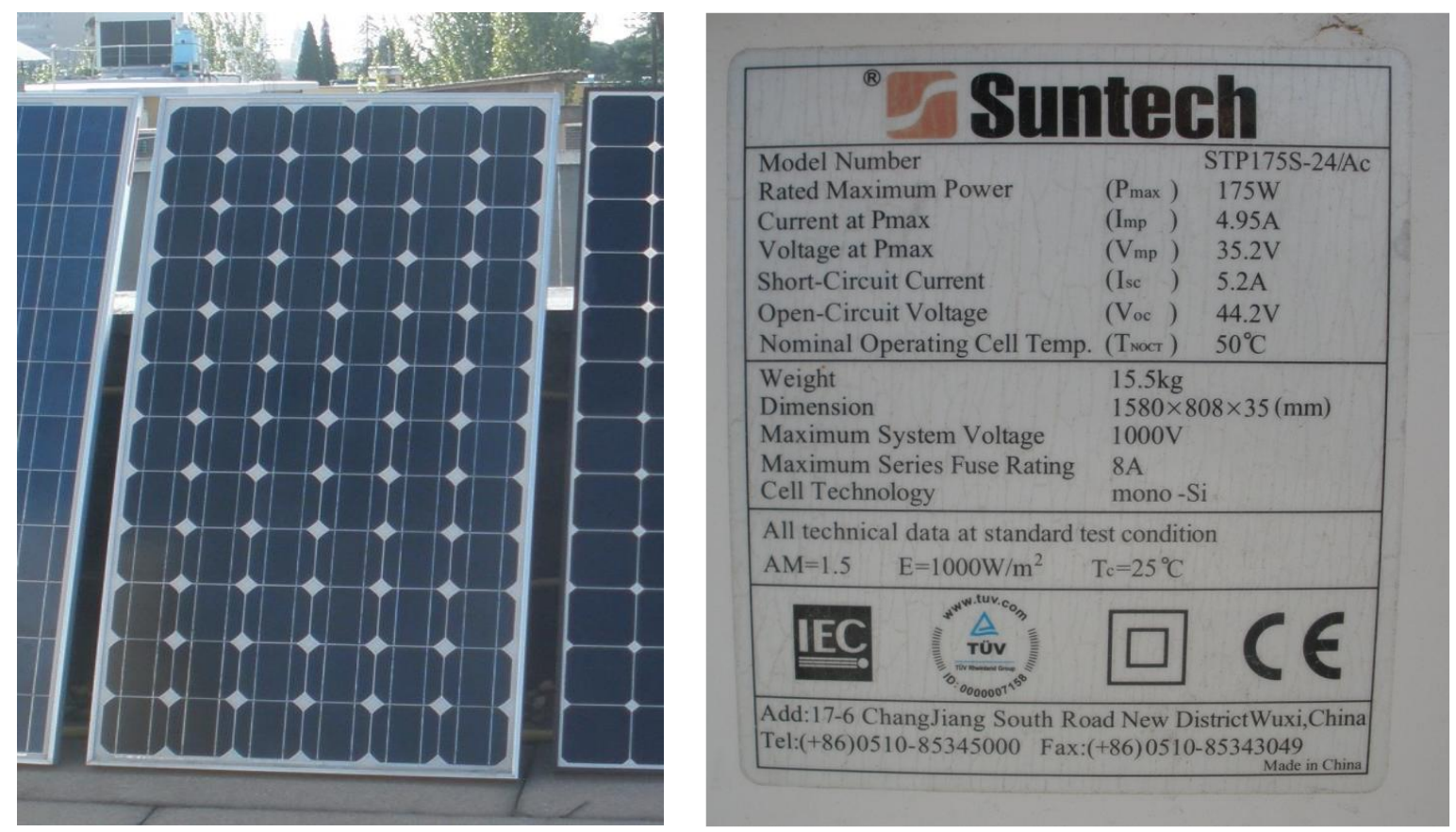

Figura 1. Módulo Fotovoltaico utilizado en el trabajo de investigación: a) Módulo Fv SUNTECH STP 175S-24/Ac, y b) Especificaciones del módulo (al reverso del panel)

Fuente: Elaboración propia, 2013.

b) Dispositivo fotovoltaico de Referencia PVPM 1000CX (Portable peak power and I-V curve measurement device (curve tracer) for photovoltaic modules): Se trata de un equipo de tecnología alemana que realiza la medición de curvas I-V de forma automática. A partir de los datos medidos, calcula parámetros característicos de las células solares: Peak Power " $\mathrm{P}_{\mathrm{pk}}$ " e Internal Series Resistance " $\mathrm{R}_{\mathrm{s}}$ ". La curva característica I-V se puede mostrar directamente en la pantalla del equipo. Después de las mediciones, los datos se almacenan automáticamente en la base de datos y por lo tanto están disponibles para trabajo en gabinete en los posteriores días al ensayo. El dispositivo puede almacenar datos de varios miles de mediciones es un dispositivo electrónico de medición que solo puede ser operado en un determinado rango de temperatura (pve Photovoltaik Engineering, 2016), razón por la que el PVPM 1000 CX no debe estar 
JOURNAL BOLIVIANO DE CIENCIAS - Vol. 17 - Número 50

ISSN: 2075-8944

Universidad Privada del Valle - Bolivia

https://doi.org/10.52428/20758944.v17i50.24

expuesto al sol por un largo periodo de tiempo. Por tanto, se debe mantener el equipo en un lugar sombreado al hacer las mediciones (Figura 2).
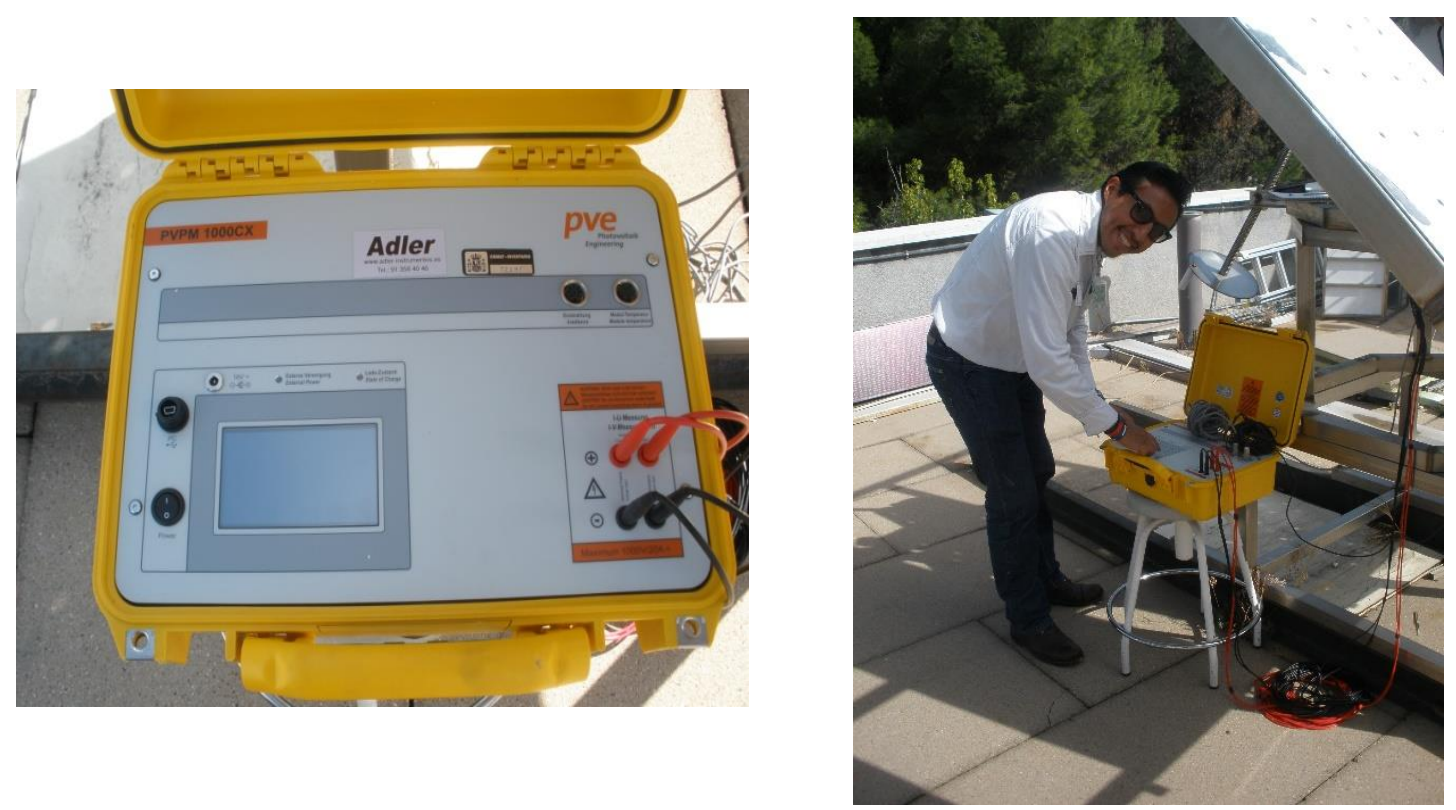

Figura 2. Dispositivo fotovoltaico de referencia para trazar curvas I-V: a) Dispositivo fotovoltaico de Referencia en lugar sombreado, y b) Trazador de curvas I-V conectado a sensor de irradiancia y al termopar para determinar la temperatura del dispositivo bajo ensayo Fuente: Elaboración propia, 2013.

c) Equipo para determinar la temperatura del dispositivo bajo (módulo Fv SUNTECH STP 175S24/Ac) ensayo utilizando el método equivalente de la temperatura de la célula (TEC): En el caso de estudio, se trata de un sensor de temperatura externo (termopar) que viene incluido con el PVPM1000CX y que debe conectarse en el puerto de entrada PT100 (Figura 3).

d) Sensor de irradiancia: Se trata de un sensor que monitoriza la irradiancia de forma instantánea en el plano de medida. Este sensor de irradiancia debe tener su cara activa coplanar con la cara activa de la muestra de ensayo (en nuestro caso el módulo). En el trabajo de investigación, el sensor de irradiancia estaba conectado con el PVPM 1000CX (Figura 4). 
JOURNAL BOLIVIANO DE CIENCIAS - Vol. 17 - Número 50

ISSN: $2075-8944$

Universidad Privada del Valle - Bolivia

https://doi.org/10.52428/20758944.v17i50.24
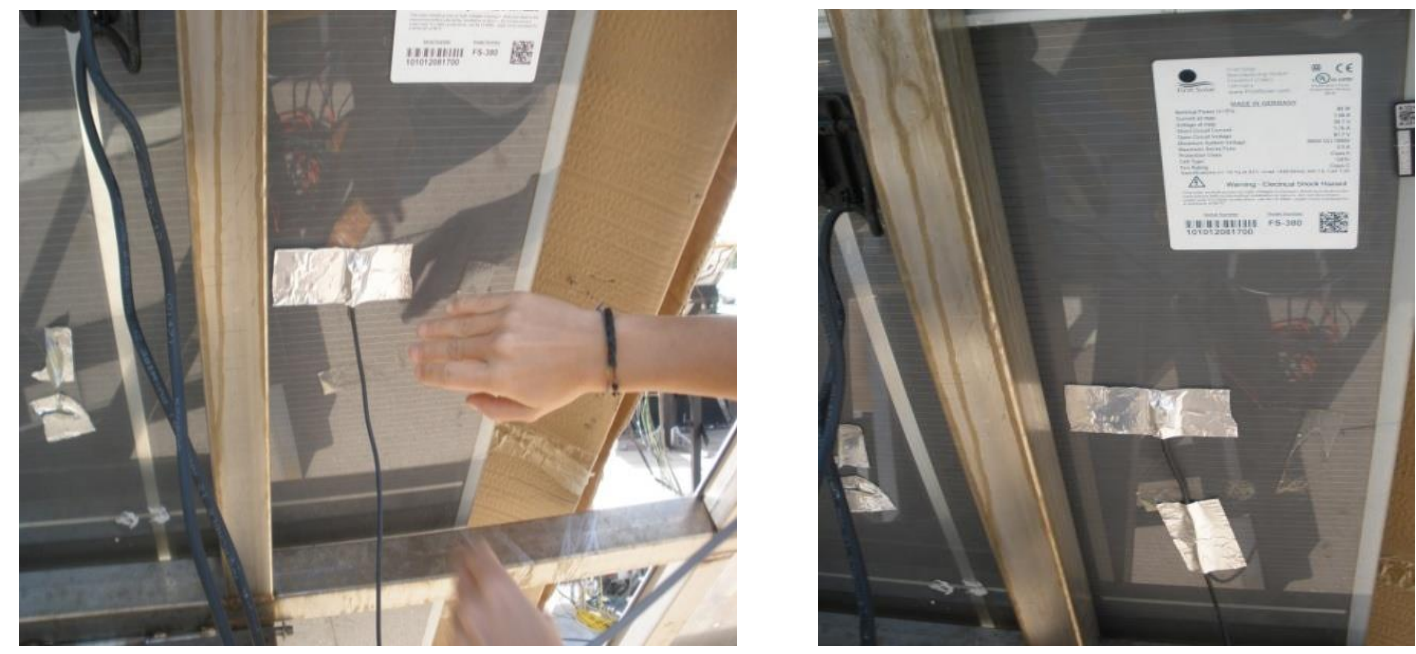

Figura 3. Sensor de Temperatura: a) Acople del sensor de temperatura (termopar) y b)

Termopar acoplado al mismo módulo

Fuente: Elaboración propia, 2013.
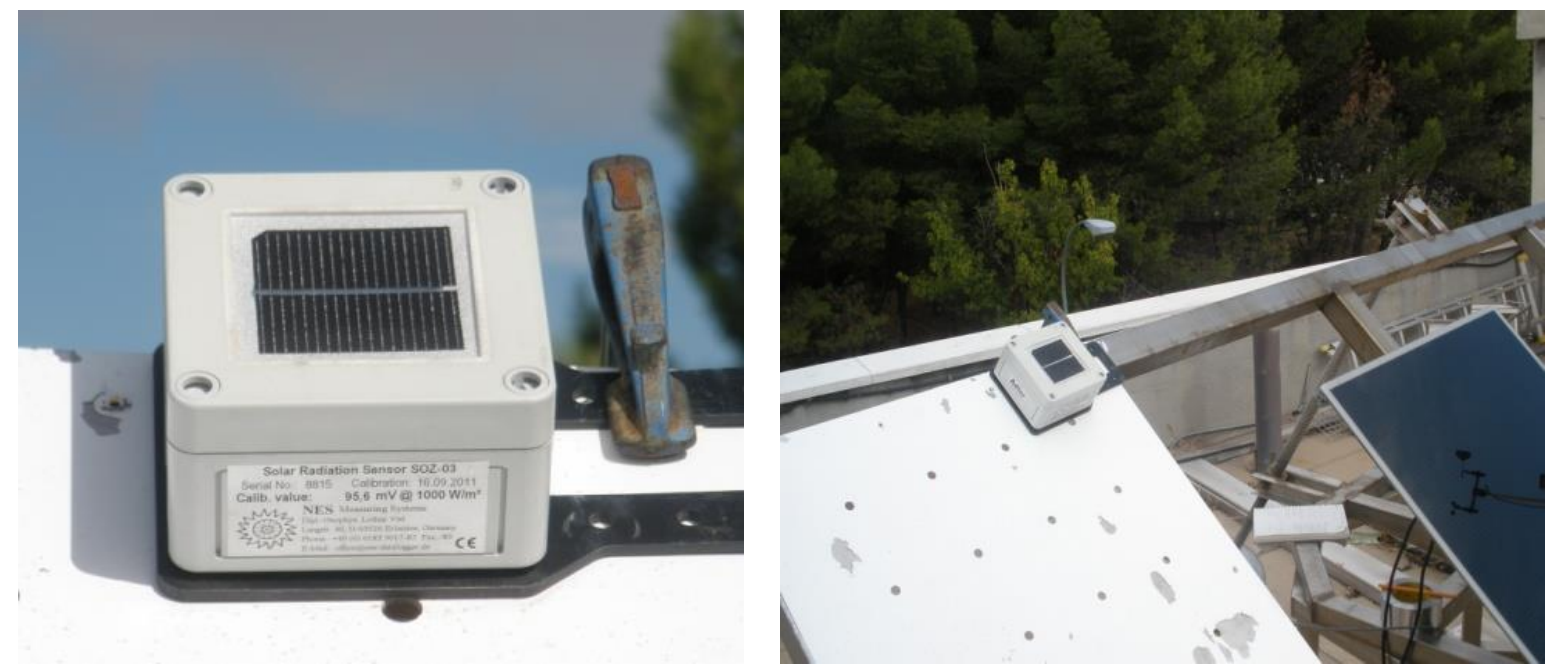

Figura 4. Sensor de Irradiancia: a) Vista del sensor de irradiancia que viene incluido en el PVPM 1000CX y b) Sensor de irradiancia ubicado cerca y en el mismo plano que el módulo a medir

Fuente: Elaboración propia, 2013. 
JOURNAL BOLIVIANO DE CIENCIAS - Vol. 17 - Número 50

ISSN: $2075-8944$

Universidad Privada del Valle - Bolivia

https://doi.org/10.52428/20758944.v17i50.24

e) Estructura de Soporte: Se trata de un sistema de seguimiento en dos ejes capaz de seguir la trayectoria del sol con una precisión de $+/-5^{\circ} \mathrm{C}$ (Figura 5).
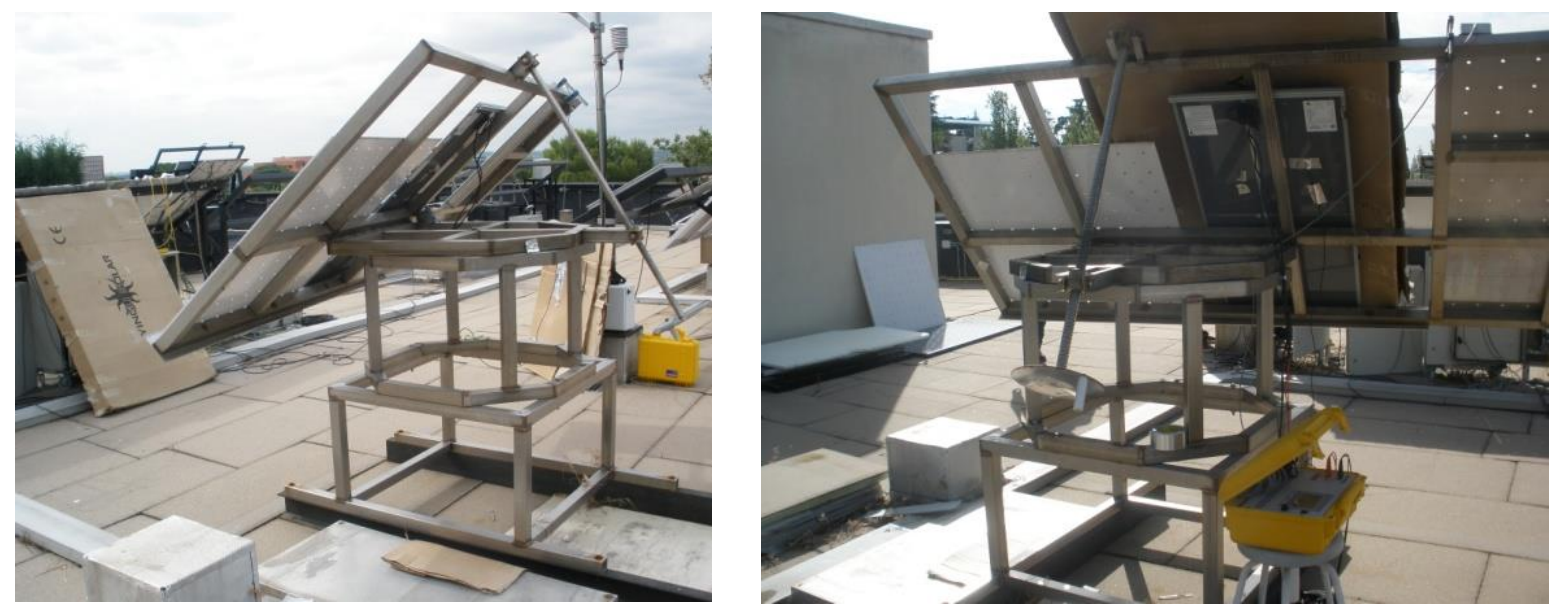

Figura 5. Estructura de soporte: a) Estructura de soporte con 2 ejes y b) Otra vista de la estructura de soporte con 2 ejes

Fuente: Elaboración propia, 2013.

\subsection{Procedimiento realizado}

El procedimiento realizado para la elaboración del presente trabajo de investigación comprendió 6 pasos:

1. Lectura de la norma de referencia IEC 60904-1:2020 (IEC, 2020).

2. Selección de un módulo fotovoltaico y obtención de la hoja de datos técnicos del fabricante para resumir en una tabla las principales características eléctricas: $\mathrm{P}_{\mathrm{MAX}}, \mathrm{I}_{\mathrm{sc}}$, $\mathrm{V}_{\text {oc }}, \mathrm{V}_{\mathrm{PMP}}$, IPMP. Incluir los valores de los coeficientes de temperatura de la corriente, la tensión y la potencia $(\alpha, \beta, \delta)$.

3. Montaje del sistema de medida de las curvas de acuerdo con la norma y las características del módulo a medir.

4. Medición de nueve curvas características del módulo en distintas condiciones de temperatura y de irradiancia utilizando el trazador PVPM 1000CX.

5. Importación de los datos obtenidos a una planilla electrónica, o programa similar para trazar las curvas IV con los datos medidos. 


\section{JOURNAL BOLIVIANO DE CIENCIAS - Vol. 17 - Número 50}

ISSN: $2075-8944$

Universidad Privada del Valle - Bolivia

https://doi.org/10.52428/20758944.v17i50.24

6. Elaborar un análisis e interpretación de los resultados obtenidos para procesar conclusiones y recomendaciones.

\subsection{Consideraciones realizadas}

Se ha montado el dispositivo de referencia (dispositivo de medida de la irradiancia) lo más cerca posible y en el mismo plano junto a la muestra (módulo fotovoltaico) en un seguidor solar de dos ejes. Ambos dispositivos deben ser perpendiculares a la radiación solar directa en un margen de $\pm 5^{\circ}$. Se han efectuado las conexiones a la instrumentación necesaria. Las Figuras 1, 2, 3, 4 y 5 muestran el procedimiento indicado.

Los elementos de medición y aquellos a ser medidos en el presente trabajo, todos con control de temperatura, han sido ajustados a los nueve controles indicados. Para el caso que no se disponga de los controles de temperatura o que no sean usados, se debe aislar el elemento de medición y aquel a ser medido, tanto del sol como del viento, esto hasta que se obtenga una temperatura uniforme con una holgura de $+/-2^{\circ} \mathrm{C}$ o la temperatura ambiente (Williams, 2012). Además, permitir que el elemento a ser medido (Módulo FV) se estabilice en cuanto a temperatura. Finalmente, preacondicionar el módulo FV a un punto debajo de la temperatura deseada y después dejar que el módulo caliente naturalmente con el ambiente. Puede existir diferencias entre el promedio de temperatura de células y el promedio de la temperatura posterior durante el calentamiento.

En la mayoría de los casos, la inercia térmica del módulo FV y del trazador de curvas, ha limitado el incremento de temperatura durante los primeros segundos por debajo de $2{ }^{\circ} \mathrm{C}$. Se ha comprobado que la temperatura de la muestra y del dispositivo de referencia son estables y se mantienen constantes en un margen de $\pm 1{ }^{\circ} \mathrm{C}$, además que la irradiancia tal como se mide con el dispositivo de referencia se mantiene constante dentro de $\pm 1 \%$ (por fluctuaciones causadas por nubes, bruma, o humo) durante el período de registro de cada serie de datos.

Para el caso de utilizar como dispositivo de referencia un piranómetro o un dispositivo de referencia no acoplado (no utilizado en la presente investigación), se realiza una medida 
JOURNAL BOLIVIANO DE CIENCIAS - Vol. 17 - Número 50

ISSN: 2075-8944

Universidad Privada del Valle - Bolivia

https://doi.org/10.52428/20758944.v17i50.24

simultánea de la irradiancia espectral utilizando el espectroradiómetro, se calcula la irradiancia efectiva para la muestra con el espectro AM 1.5, utilizando la respuesta espectral. La medida deberá realizarse en un día despejado y asoleado.

\subsection{Datos técnicos del prototipo de ensayo}

En la Tabla 1 se muestra las características técnicas del módulo fotovoltaico (de industria China) utilizado en el presente trabajo de investigación.

\begin{tabular}{lll}
\hline Manufacturer & & SUNTECH \\
\hline Number model & & STP 175s-24/Ac \\
\hline Maximum power & Pmáx & $175 \mathrm{~W}$ \\
\hline Max. power voltage & Vmp & $35,8 \mathrm{~V}$ \\
\hline Max. power current & Imp & $4,9 \mathrm{~A}$ \\
\hline Open circuit voltage & Voc & $44,7 \mathrm{~V}$ \\
\hline Short circuit current & Isc & $5,23 \mathrm{~A}$ \\
\hline Temperature coeff. of $\mathbf{P}_{\max }$ & $\boldsymbol{\delta}$ & $-0,48$ \\
\hline Temperature coeff. of $\mathbf{V}_{\mathbf{o c}}$ & $\boldsymbol{\beta}$ & $-0,34$ \\
\hline Temperature coeff. of $\mathbf{I}_{\mathbf{s c}}$ & $\boldsymbol{\alpha}$ & 0,017 \\
\hline Module Efficiency & & $14.1 \%$ \\
\hline Technology & & SILICIO \\
\hline
\end{tabular}

Tabla 1. Características técnicas módulo FV

Fuente: Energy Matters, 2008.

\section{RESULTADOS Y DISCUSIÓN}

Para el presente trabajo de investigación, utilizando como espécimen al módulo fotovoltaico SUNTECH STP 175S-24/Ac, se han realizado cuatro mediciones de irradiación solar (a 596 $\mathrm{W} / \mathrm{m}^{2}, 727 \mathrm{~W} / \mathrm{m}^{2}, 784 \mathrm{~W} / \mathrm{m}^{2}, 857 \mathrm{~W} / \mathrm{m}^{2}$ ) a una temperatura constante de $51,4{ }^{\circ} \mathrm{C}$; y por otro lado se realizaron cinco mediciones de temperatura $\left(\right.$ a $38{ }^{\circ} \mathrm{C}, 40{ }^{\circ} \mathrm{C}, 44,9{ }^{\circ} \mathrm{C}, 49,65{ }^{\circ} \mathrm{C}, 53,4{ }^{\circ} \mathrm{C}$ ) a una irradiancia constante de $857 \mathrm{~W} / \mathrm{m}^{2}$. Los resultados obtenidos se muestran en la Tabla 2 y en la Tabla 3. 
La forma de las curvas, obtenidas con el trazador de curvas PVPM 1000CX, es la esperada (Figuras 6 y 7). Cuando la tensión entre sus terminales es baja, la intensidad de corriente que entrega es aproximadamente constante; mientras que, a mayor tensión la corriente se desploma rápidamente.

En esta curva destacan dos puntos especiales (Martín et al., 2007): a) En primer lugar, el punto de la curva que tiene tensión cero, en el que la intensidad de corriente en estas condiciones se llama Intensidad de cortocircuito $\left(\mathrm{I}_{\mathrm{sc}}\right)$. La corriente de cortocircuito es la intensidad máxima de la corriente que se puede obtener de un panel. Se mide con un amperímetro conectando entre los bornes del panel sin ninguna resistencia adicional. Al no existir resistencia alguna al paso de la corriente, la caída de potencial es cero. $\mathrm{I}_{\mathrm{sc}}$ constituye la máxima corriente que puede obtenerse de una célula solar y es directamente proporcional a la luz disponible.

b) El segundo punto importante de la curva se consigue dejando en circuito abierto el módulo, eso reduce a cero la corriente y la tensión correspondiente se llama Tensión de circuito abierto $\left(\mathrm{V}_{\mathrm{oc}}\right)$; es el voltaje máximo que se podría medir con un voltímetro, en condiciones de circuito abierto, su valor se relaciona con el número de células que tiene el módulo conectadas en serie. En los módulos fotovoltaicos ordinarios de silicio (como es el caso del presente estudio) cada una de esas células contribuye aproximadamente con $0,6 \mathrm{~V}$.

\subsection{Mediciones a temperatura constante}

Las mediciones obtenidas con el trazador de curvas PVPM 1000CX a temperatura constante de $51,4{ }^{\circ} \mathrm{C}$ (en el módulo FV) para cuatro irradiancias diferentes se muestran en la Tabla 2 y la Figura 6. 
JOURNAL BOLIVIANO DE CIENCIAS - Vol. 17 - Número 50

ISSN: $2075-8944$

Universidad Privada del Valle - Bolivia

https://doi.org/10.52428/20758944.v17i50.24

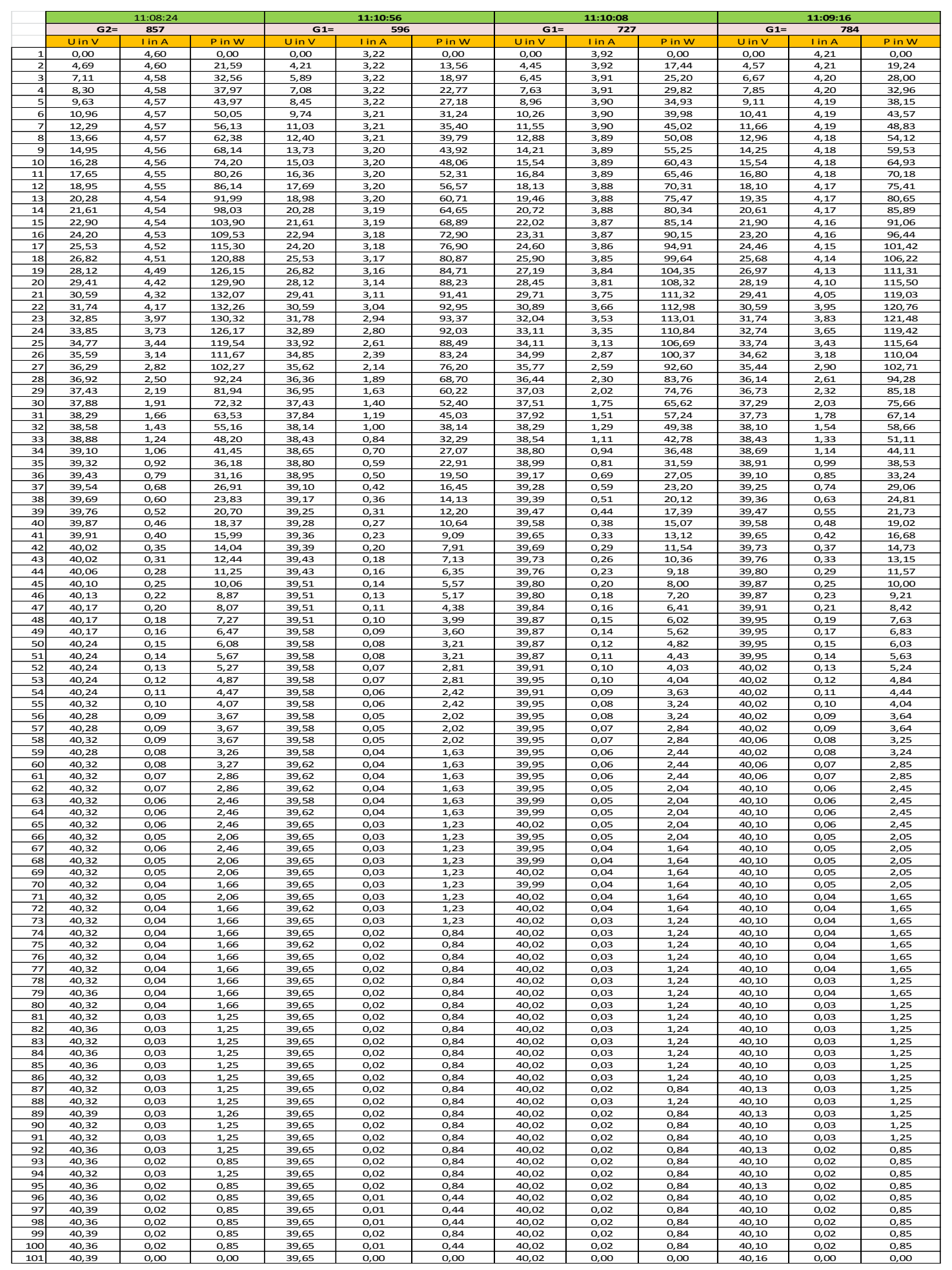

Tabla 2. Tabla de mediciones obtenidas a temperatura constante de $51,4^{\circ} \mathrm{C}$ para cuatro

irradiancias solares

Fuente: Elaboración propia, 2013. 
JOURNAL BOLIVIANO DE CIENCIAS - Vol. 17 - Número 50

ISSN: $2075-8944$

Universidad Privada del Valle - Bolivia

https://doi.org/10.52428/20758944.v17i50.24

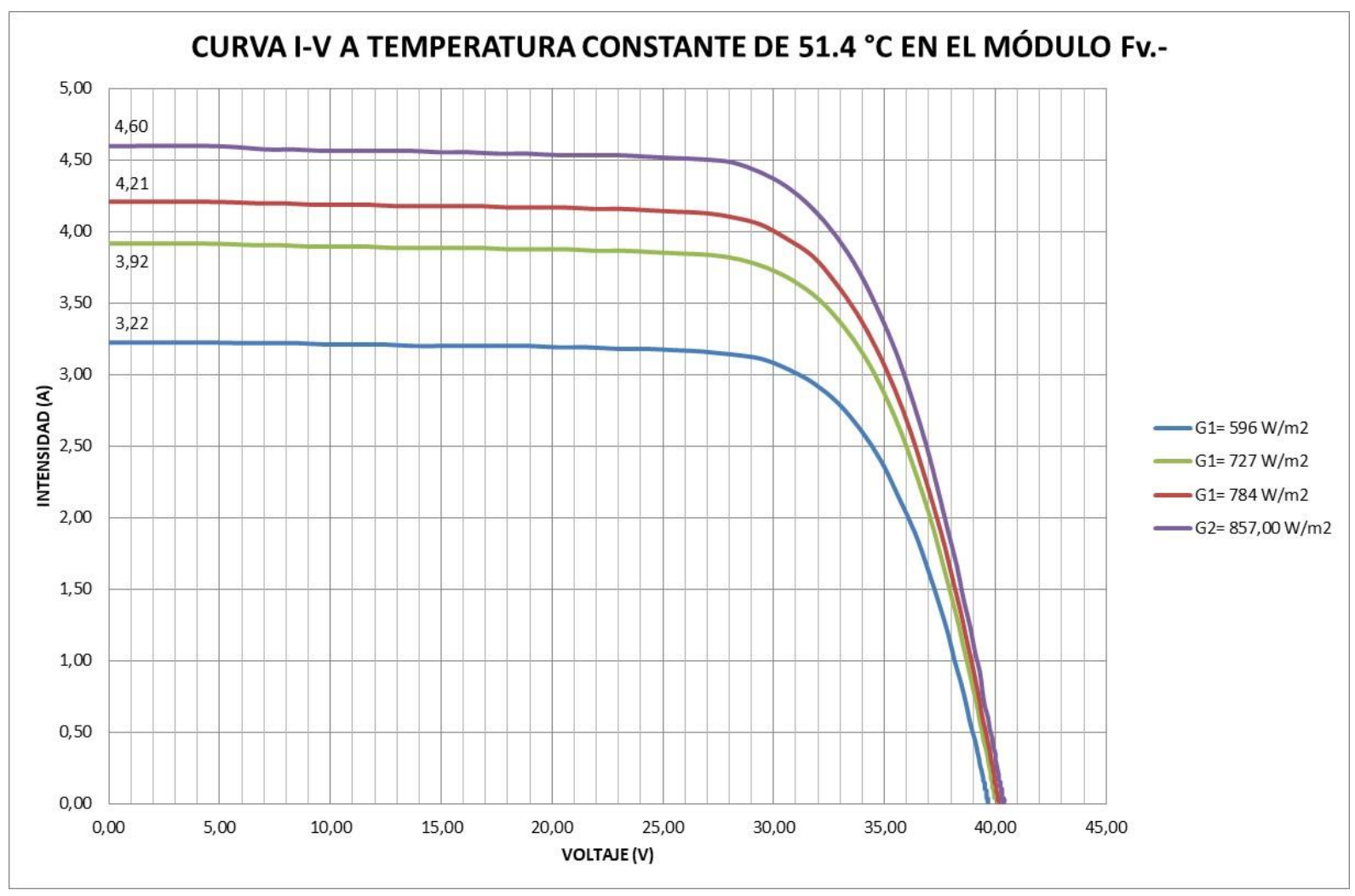

Figura 6. Curvas I-V para temperatura constante

Fuente: Elaboración propia, 2013.

Una vez analizados los resultados, se observa que las distintas irradiancias solares han generado que la curva cambie en lo que respecta a la intensidad de corriente "I", pero manteniendo constante (o con una ligera variación) la tensión de voltaje "V". En ningún caso, de las 4 mediciones realizadas, se ha sobrepasado los valores de " $\mathrm{I}_{\mathrm{sc}}$ " y " $\mathrm{V}_{\mathrm{oc}}$ " del módulo fotovoltaico correspondientes a las condiciones estándar (Figuras 8 y 9). Por lo tanto, se observa que, bajo cualquier variación de irradiancia solar, que puede ser ocasionada por nubes u otros factores, el módulo fotovoltaico ensayado no llega a sus condiciones más desfavorables de operación. En resumen, la tensión de circuito abierto " $\mathrm{V}_{\mathrm{oc}}$ " apenas varía, pero la intensidad de corto circuito "Isc" se reduce proporcionalmente a la reducción de irradiancia solar para cada caso. Todo el comportamiento que ha sido monitoreado y analizado es una consecuencia de que al módulo le llega menos energía. 
JOURNAL BOLIVIANO DE CIENCIAS - Vol. 17 - Número 50

ISSN: 2075-8944

Universidad Privada del Valle - Bolivia

https://doi.org/10.52428/20758944.v17i50.24

\subsection{Mediciones a Irradiación solar constante}

Las mediciones obtenidas con el trazador de curvas PVPM 1000CX en el ensayo a irradiación solar constante de $857 \mathrm{~W} / \mathrm{m}^{2}$ para cuatro temperaturas diferentes se muestran en la Tabla 3 y en la Figura 7.

Una vez analizados los resultados, se observa que las distintas variaciones de temperatura han generado que la curva cambie en lo que respecta al voltaje "V", pero manteniendo constante la intensidad de corriente "I". En ningún caso se ha sobrepasado los valores de "I $\mathrm{sc}_{\text {" }}$ y "V $\mathrm{V}_{\mathrm{oc}}$ " del módulo fotovoltaico correspondientes a las condiciones estándar. De manera que el rol de la variación de temperatura en el módulo fotovoltaico ensayado, que puede ser ocasionado por la ubicación geográfica, el clima u otros factores, no llega a alterar sus condiciones más desfavorables de operación.

Si bien, en el ensayo a condiciones estándar se exige que la temperatura en las células solares del módulo fotovoltaico sea de $25{ }^{\circ} \mathrm{C}$, durante la fase de operación del módulo fotovoltaico, la temperatura será distinta a $25{ }^{\circ} \mathrm{C}$, en la mayoría de los casos. La Figura 7 muestra que, si la temperatura es mayor, la intensidad de cortocircuito "I $\mathrm{sc}_{\mathrm{sc}}$ aumenta ligeramente; pero la tensión de circuito abierto "Voc" disminuye más acusadamente. Como consecuencia, esto va a conducir a una reducción de la potencia máxima del módulo fotovoltaico $\left(\mathrm{P}_{\mathrm{M}}\right)$ : Un 0,5 \% de reducción en la potencia por cada grado por encima de $25{ }^{\circ} \mathrm{C}$ que aumente la temperatura del módulo fotovoltaico (Ente Regional de la Energía de Castilla y León [EREN], 2004). Las mediciones obtenidas a irradiancia constante de $857 \mathrm{~W} / \mathrm{m}_{2}$ para cinco temperaturas diferentes se muestran en la Figura 7 y la Tabla 3. 
JOURNAL BOLIVIANO DE CIENCIAS - Vol. 17 - Número 50

ISSN: 2075-8944

Universidad Privada del Valle - Bolivia

https://doi.org/10.52428/20758944.v17i50.24

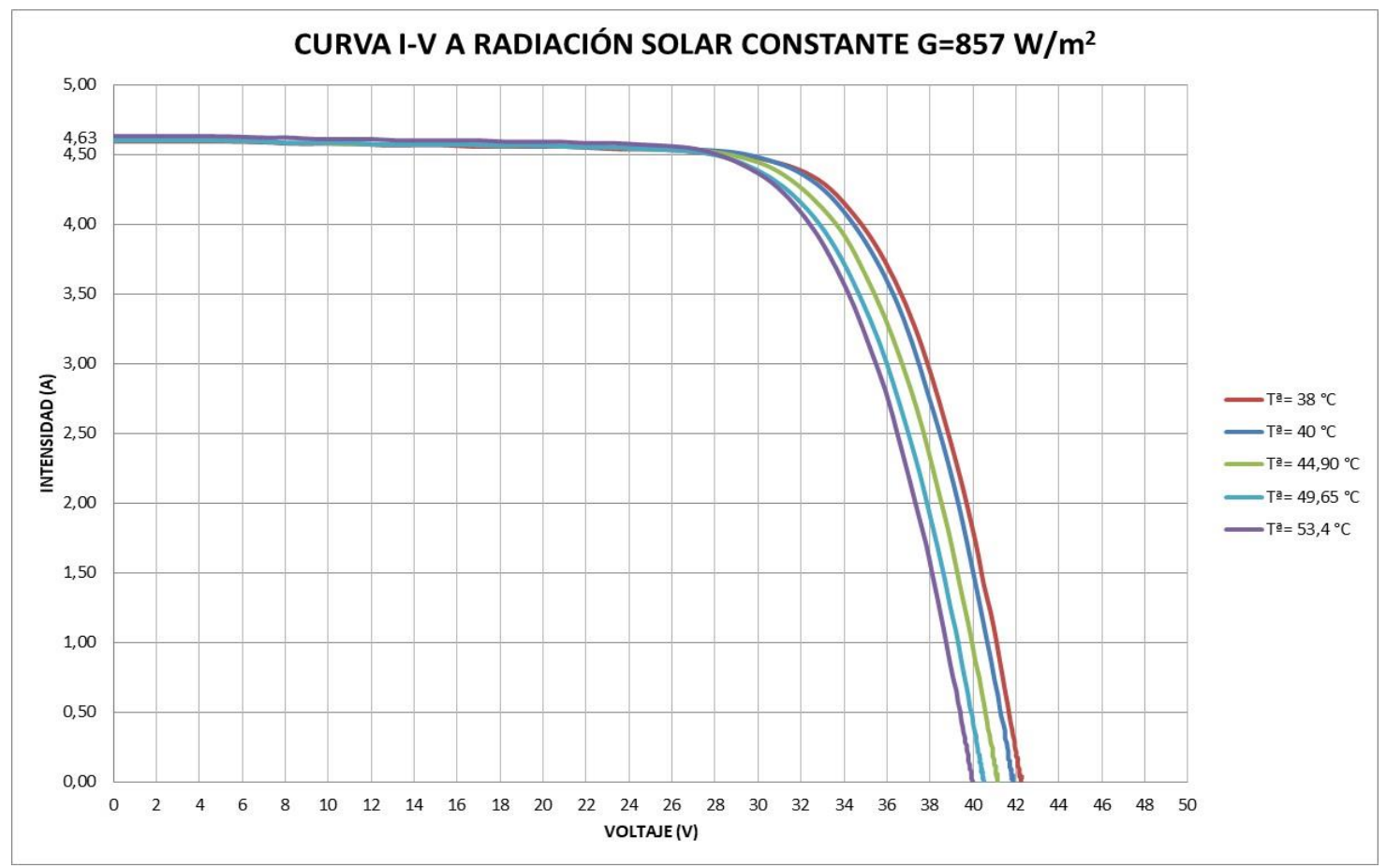

Figura 7. Curvas I-V medidas a irradiancia solar constante de $857 \mathrm{~W} / \mathrm{m}^{2}$ Fuente: Elaboración propia, 2013.

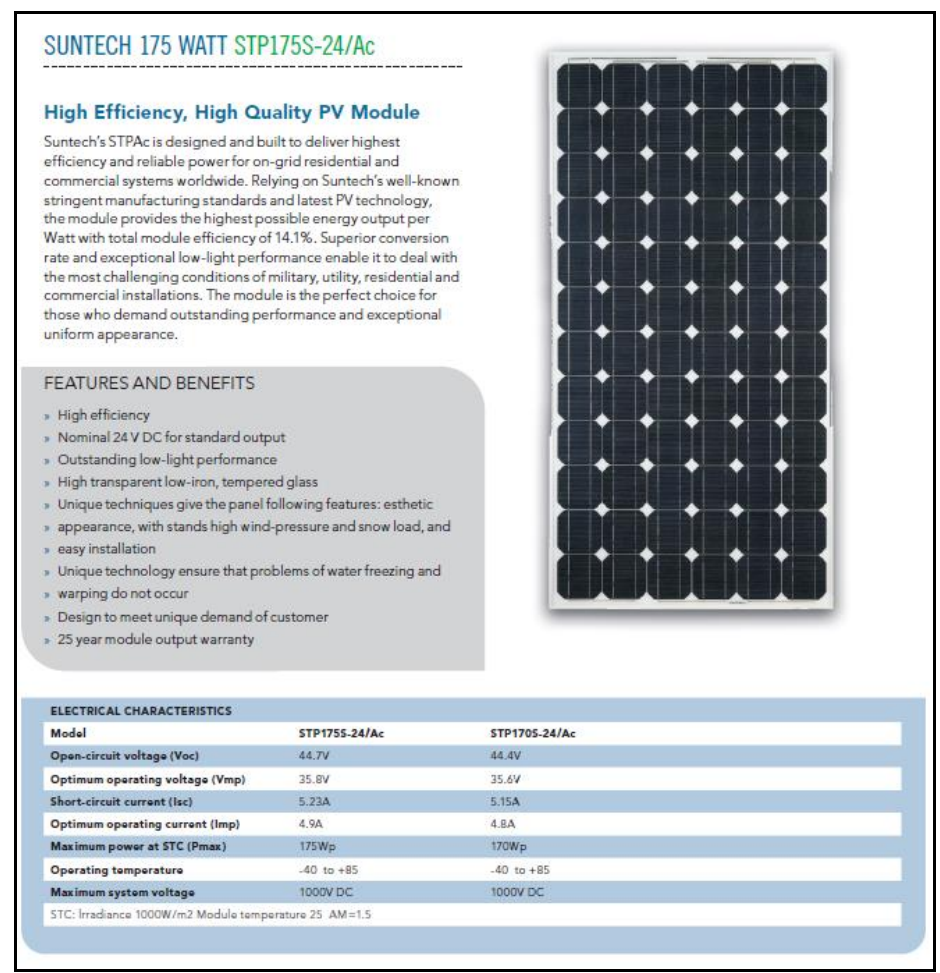

Figura 8. Hoja de especificaciones técnicas del módulo fotovoltaico medido Fuente: Energy Matters, 2008. 
JOURNAL BOLIVIANO DE CIENCIAS - Vol. 17 - Número 50

ISSN: $2075-8944$

Universidad Privada del Valle - Bolivia

https://doi.org/10.52428/20758944.v17i50.24

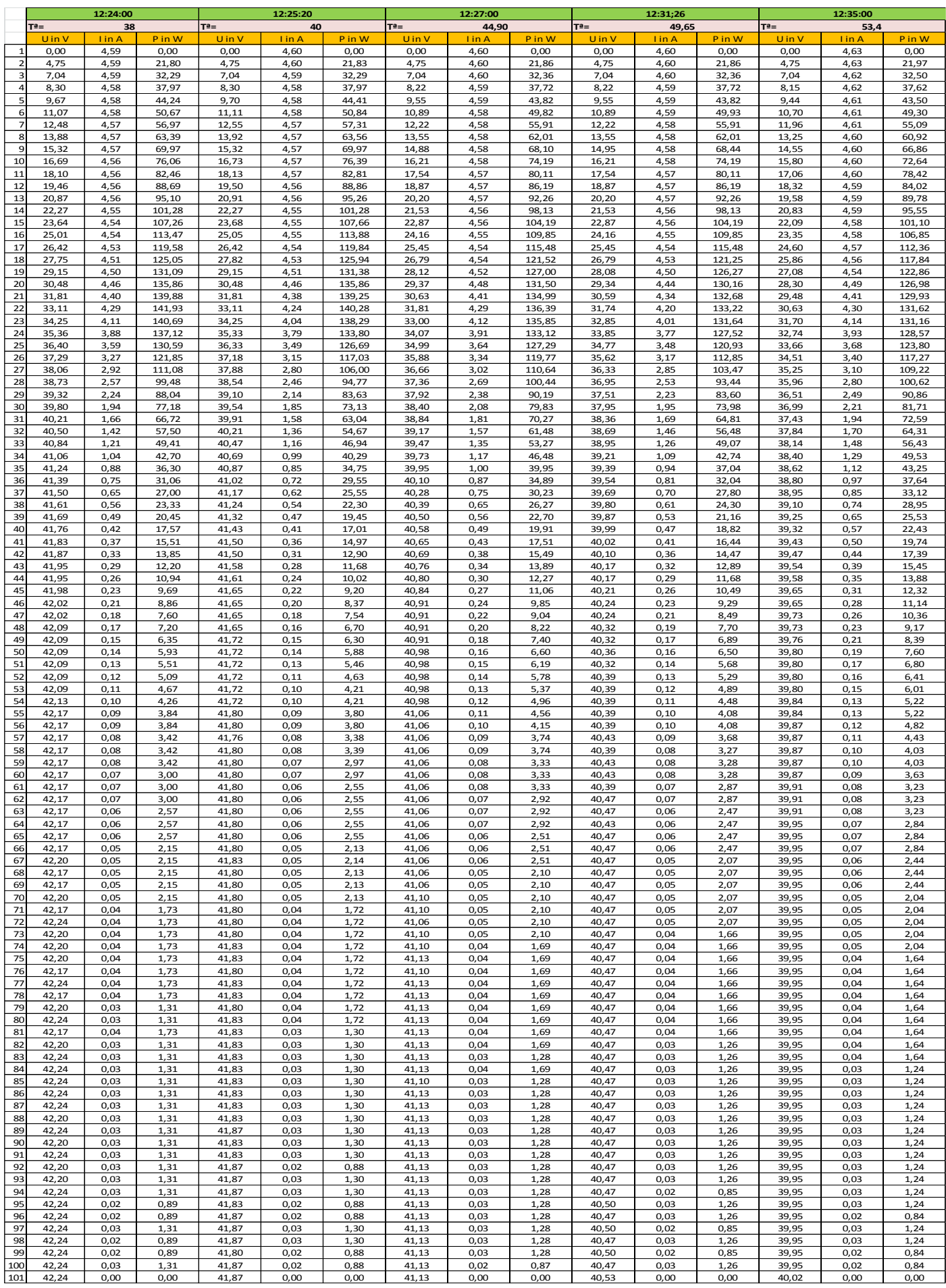

Tabla 3. Tabla de mediciones obtenidas a irradiancia constante de $857 \mathrm{~W} / \mathrm{m} 2$ para cinco

temperaturas diferentes del módulo fotovoltaico

Fuente: Elaboración propia, 2013. 
JOURNAL BOLIVIANO DE CIENCIAS - Vol. 17 - Número 50

ISSN: $2075-8944$

Universidad Privada del Valle - Bolivia

https://doi.org/10.52428/20758944.v17i50.24

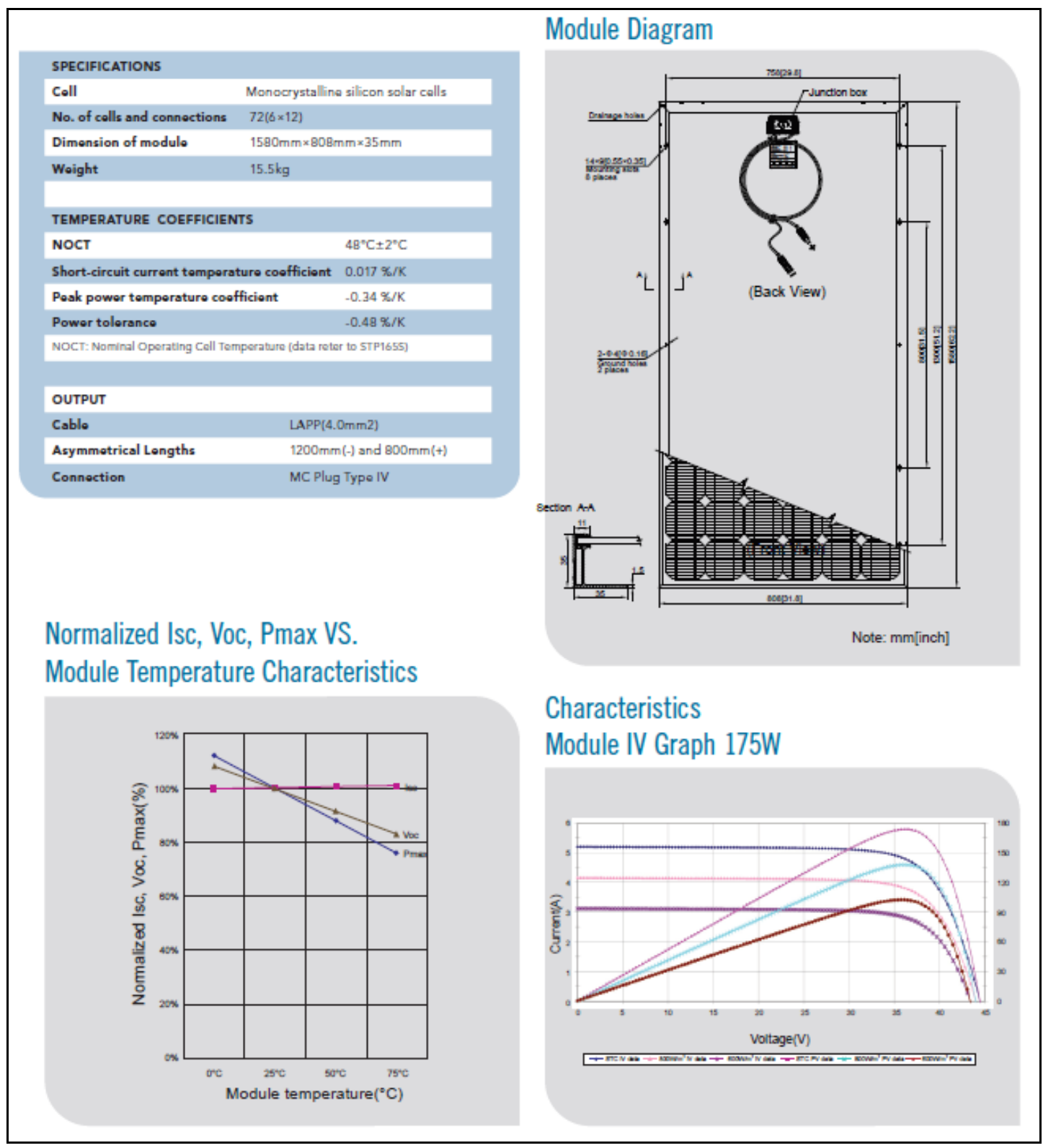

Figura 9. Hoja de especificaciones técnicas del módulo fotovoltaico medido

Fuente: Energy Matters, 2008.

\section{CONCLUSIONES}

Las cuatro mediciones realizadas al módulo fotovoltaico SUNTECH STP 175S-24/Ac a temperatura constante de $51,4^{\circ} \mathrm{C}$ con el trazador de curvas PVPM $1000 \mathrm{CX}$, proporcionan cuatro curvas I-V en las que la intensidad de cortocircuito ( $\mathrm{I}_{\mathrm{sc}}$ ) varía entre 3,22 A y 4,60 A (Figura 6), la 


\section{JOURNAL BOLIVIANO DE CIENCIAS - Vol. 17 - Número 50}

ISSN: $2075-8944$

Universidad Privada del Valle - Bolivia

https://doi.org/10.52428/20758944.v17i50.24

tensión de circuito abierto $\left(\mathrm{V}_{\mathrm{oc}}\right)$ varía entre 39,62 V y 40,39 V (Figura 6) y la potencia entregada por el módulo fotovoltaico ha registrado un valor máximo de 132,36 W (Tabla 2).

Las cinco mediciones realizadas al módulo FV a irradiación solar constante de $857 \mathrm{~W} / \mathrm{m}^{2}$ con el trazador de curvas PVPM $1000 \mathrm{CX}$, proporcionan cinco curvas I-V en las que la intensidad de cortocircuito ( $\mathrm{I}_{\mathrm{sc}}$ ) varía de 4,59 A - 4,60 A (Figura 7), la tensión de circuito abierto ( $\mathrm{V}_{\mathrm{oc}}$ ) varía de 40,02 V - 41,87 V (Figura 7) y la potencia entregada por el módulo fotovoltaico ha registrado un valor máximo de 141,93 W (Tabla 3).

Los resultados obtenidos en el presente trabajo de investigación demuestran que, sometiendo el módulo fotovoltaico SUNTECH STP 175s-24/Ac a nueve condiciones experimentales de operación, como la variación de la irradiancia solar y la temperatura del módulo fotovoltaico, en las mediciones realizadas, estas variaciones no llegan a superar en la intensidad de cortocircuito $\left(\mathrm{I}_{\mathrm{sc}}=5,23 \mathrm{~A}\right)$ y la tensión de circuito abierto $\left(\mathrm{V}_{\mathrm{oc}}=44,7 \mathrm{~V}\right)$, correspondientes a las condiciones estándar de trabajo del módulo fotovoltaico objeto del presente estudio (Figuras 8 y 9).

También, se ha observado que estas condiciones experimentales provocan una disminución en la potencia máxima entregada del módulo fotovoltaico ( $\mathrm{P}_{\mathrm{M}}$ ) (Tabla 2 y Tabla 3), información que puede ser útil a la hora de diseñar sistemas fotovoltaicos. En principio, el módulo fotovoltaico puede trabajar en cualquier punto de su curva I-V.

Las mediciones realizadas son desfavorables en el caso de la temperatura del módulo fotovoltaico, ya que en las nueve mediciones los módulos se encuentran a una temperatura por encima de $25^{\circ} \mathrm{C}$. En el caso de la radiación solar, las mediciones están por debajo de $1000 \mathrm{~W} / \mathrm{m}^{2}$. En todas las mediciones, la potencia máxima entregada del módulo FV no ha llegado a superar al $81 \%$ de la potencia en condiciones estándar $(175 \mathrm{~W})$, que en la práctica ocasiona que la producción final del módulo disminuya.

Las curvas I-V obtenidas del módulo fotovoltaico SUNTECH STP 175s-24/Ac a través del trazador de curvas PVPM 1000CX, permiten calcular la potencia instantánea $\left(\mathrm{P}_{\mathrm{M}}\right)$ en función de 


\section{JOURNAL BOLIVIANO DE CIENCIAS - Vol. 17 - Número 50}

ISSN: $2075-8944$

Universidad Privada del Valle - Bolivia

https://doi.org/10.52428/20758944.v17i50.24

las condiciones de operación (irradiancia solar, temperatura de célula y respuesta espectral principalmente) que varían a lo largo del día. Las mediciones realizadas en el presente trabajo de investigación se realizaron en Madrid-España; sin embargo, se ha intentado mostrar el potencial que existe en un laboratorio de fotovoltaica al contar con los dispositivos de medición utilizados en el presente trabajo; que bien podrían servir para analizar patrones de sombreado en sistemas FV para edificios.

En la realidad, no es posible mantener la temperatura y la irradiación solar constantes, siempre existirá variación. Sin embargo, con el presente trabajo de investigación se muestra que la manipulación de estos parámetros disminuye la potencia máxima entregada del módulo FV, parámetro importante en el diseño de sistemas FV.

La radiación solar en el Departamento de Chuquisaca es más que suficiente para poder satisfacer con garantía las demandas de electricidad de un importante número de usuarios, viviendas, explotaciones agrícolas y ganaderas, bombeos, telecomunicación y señalización, alumbrado de viales y/o suministro de energía eléctrica a la red de distribución, de manera económica, promoviendo la coparticipación y corresponsabilidad en la utilización de una energía menos contaminante.

\section{AGRADECIMIENTOS}

Agradecemos a nuestro tutor Ph.D. Nuria Martín Chivelet (Laboratorio de Fotovoltaica, C.I.E.M.A.T.) por el asesoramiento realizado en el trabajo.

\section{REFERENCIAS}

Abella, M. (2001). Sistemas fotovoltaicos. Madrid: S.A.P.T. ISBN 84-86913-09-8

Krenzinger, A., Bühler, A., Gasparín, F. (2009). Aparato para medir curvas características de módulos fotovoltaicos con radiación natural. XVI Simposio Peruano de Energia Solar, Arequipa, Perú. 
Martín, N., Alonso-García, M., Chenlo, F., Sánchez-Friera, P. (2007). Electrical and thermal characterisation of PV modules under partial shadowing. 23rd European Photovoltaic Solar Energy Conference, Valencia, España.

International Electrotechnical Commision (IEC) (2020). 60904-1:2020 Photovoltaic devices Part 1: Measurement of photovoltaic current-voltage characteristics. IEC.

Ente Regional de la Energía de Castilla y León (EREN) (2004). Energía Solar fotovoltaica. Gráficas Celarayn. ISBN 84-9718-259-6

Collado, E. (13 de Julio de 2015). Los efectos de la temperatura en la producción de las instalaciones fotovoltaicas. Energías renovables: El periodismo de las energías limpias. Recuperado de https://www.energias-renovables.com/fotovoltaica/los-efectos-de-latemperatura-en-la-20150713

Energy Matters (2008). We make solar power simple. Canberra, Australia.: Digital Eagles. Recuperado de https://www.energymatters.com.au/images/suntech/suntech175w\%20up.pdf

pve Photovoltaik Engineering. (2016). Präzise I-U-Kennlinienmessgeräte für die PhotovoltaikBranche. Iserlohn, Alemania: PV-Engineering GmbH. Recuperado de https://www.pvengineering.de/fileadmin/user_upload/Produkte/PVPM1000CX/pvpm1000cxdatasheet_01.pdf

Williams, R. M. (2012). Análisis del Comportamiento de Módulos Fotovoltaicos. Diagnóstico, modelado Matemático de curvas I-V y P-V, y construcción de Prototipo de Trazador de curvas I- $V$ (Tesis de maestría). Universidad de Braganza, Portugal.

Fuentes de financiamiento: El presente trabajo ha sido financiado por la Fundación Simón I. Patiño como parte de la Maestría en Energías Renovables y eficiencia energética en la Universidad de Salamanca, España gestión 2012-2013.

Declaración de conflicto de intereses: Los autores declaran que no tienen ningún conflicto de interés. 
JOURNAL BOLIVIANO DE CIENCIAS - Vol. 17 - Número 50

ISSN: 2075-8944

Universidad Privada del Valle - Bolivia

https://doi.org/10.52428/20758944.v17i50.24

Copyright (c) 2021 Juan Alfredo Torrico Bravo. María Gullón Urbano.

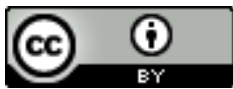

Este texto está protegido por una licencia Creative Commons 4.0.

Usted es libre para Compartir — copiar y redistribuir el material en cualquier medio o formato-y Adaptar el documento — remezclar, transformar y crear a partir del material - para cualquier propósito, incluso para fines comerciales, siempre que cumpla la condición de:

Atribución: Usted debe dar crédito a la obra original de manera adecuada, proporcionar un enlace a la licencia, e indicar si se han realizado cambios. Puede hacerlo en cualquier forma razonable, pero no de forma tal que sugiera que tiene el apoyo del licenciante o lo recibe por el uso que hace de la obra.

$\underline{\text { Resumendelicencia - Textocompletodelalicencia }}$ 